J o u r n a l of
Mathematics
and Applications
JMA No 40, pp 21-36 (2017)

\title{
On Regulated Functions
}

\author{
Józef Banaś and Mariola Kot
}

\begin{abstract}
The main purpose of this review article is to present the concept of a regulated function and to indicate the connection of the class of regulated functions with other significant classes of functions. In particular, we give a characterization of regulated functions in terms of step functions and we show that the linear space of regulated functions forms a Banach space under the classical supremum norm.
\end{abstract}

AMS Subject Classification: 26A45, 26A15.

Keywords and Phrases: Monotonic function; Function of bounded variation; Step function; Regulated function; Banach space.

\section{Introduction}

The paper is dedicated to present some basic facts concerning the so-called regulated functions. The class of those functions is very important in the theory of functions of a real variable and is especially exploited in the description and characterization of a lot of classes of functions of generalized bounded variation (cf. [1]). If is worthwhile mentioning that instead of the term "regulated function" we use also sometimes the term "regular function" [1].

It seems that the concept of a regulated function was introduced by G. Aumann in his monograph [2]. In that monograph we can find the proof of the fact that the space of regulated functions forms a Banach space but the presented proof of this fact seems to be complicated and a bit incomprehensible. The excellent proof of the mentioned fact was given in the famous book of J. Dieudonné [3]. Our presentation of the theory of regulated functions is closely patterned on the mentioned book.

Nevertheless, the proof given in [3] contains a few gaps and errors which will be improved and completed in the present paper.

As we mentioned previously the concept of a regulated function is used in investigations concerning a few classes of functions of generalized bounded variation (cf. $[5,8,9]$, for example). A comprehensive presentation of several classes of functions of generalized bounded variation and their connections with the class of regulated functions was presented in the book [1].

COPYRIGHT (c) by Publishing House of Rzeszów University of Technology P.O. Box 85, 35-959 Rzeszów, Poland 
Let us also indicate that the class of regulated functions was also used in the study of stochastic integral equations [6]. Some investigations of regulated functions were also conducted in the paper [4] in connection with the description of classes of functions which are relatively compact in the space of regulated functions. On the other hand it seems that the results obtained in that paper are not entirely satisfactory from the view point of possible applications.

The paper has a review character and it can be viewed as an introduction to further study of some problems related to the theory of regulated functions. The details will appear elsewhere.

\section{Auxiliary facts}

The basic tool used in the paper is the concept of a metric space. Thus, let us denote by $(X, d)$ a metric space. If $Y$ is a nonempty subset of $X$ then it can be regarded as a metric subspace of the space $X$ with the metric induced by $d$.

For further purposes we will denote by $B(x, r)$ the open ball (in the metric space $X$ ) with the center at $x$ and with radius $r$, respectively.

Throughout the paper we will use the standard concepts and notation of the theory of metric space (cf. [3, 10]). For example, if A is a subset of the metric space $X$ then we denote by $\bar{A}$ its closure. Obviously, if $A=\bar{A}$ then $A$ is called a closed set. If $Y$ is a subspace of the metric space $X$ and $A$ is a subset of $Y$ such that $\bar{A}=Y$, then we say that $A$ is dense in $Y$. Moreover, in the standard way we define the concept of a relatively compact and compact set in the metric space $X[3,10]$.

Now, we recall a few classical facts which will be used in our study [3].

Theorem 2.1. Let $(X, d)$ be a complete metric space. Then any nonempty, closed subset of the space $X$ is a complete subspace of $X$.

Theorem 2.2. Let $(X, d)$ be a metric space and let $A$ be a nonempty subset of $X$ such that $A$ forms a complete subspace of $X$. Then the set $A$ is closed.

In what follows we will discuss the concept of an isolated point and an isolated set [11]. Namely, if $(X, d)$ is a metric space and $A$ is a subset of $X$ then a point $x \in A$ is called an isolated point of $A$ if it is not an accumulation point of $A$ i.e., there exists $r>0$ such that $B(x, r) \cap A=\{x\}$.

A subset $A$ of the metric space $X$ is said to be an isolated set if each point of $A$ is an isolated point of the set $A$.

We have the following theorem.

Theorem 2.3. The set of all isolated points of a set $A$ is an isolated set.

Proof. The proof requires the standard reasoning. Namely, denote by is $A$ the set of all isolated points of the set $A$. Let $y \in i s A$. Then there exists $r>0$ such that $B(y, r) \cap A=\{y\}$. Hence we infer that the ball $B(y, r)$ does not contain points of the set $i s A$, except the point $y$. Indeed, it is a simple consequence of the fact that each point of the set $i s A$ belongs to the set $A$. On the other hand the ball $B(y, r)$ contains only one point of the set $A$, the point $y$. Thus $y$ is an isolated point of the set $i s A$. 
For our further purposes the next theorem will be essential.

Theorem 2.4. Let $(X, d)$ be a separable metric space. Then every isolated subset $A$ of the space $X$ is at most countable. $[11])$.

In order to make the paper self-contained we give the proof of this theorem (cf.

Proof. Let $W=\left\{y_{1}, y_{2}, \ldots\right\}$ be an at most countable dense subset of $X$. For arbitrarily fixed point $x \in A$ denote by $r_{x}$ a positive number such that $B\left(x, r_{x}\right) \cap A=\{x\}$. Next, for an arbitrary $x \in A$ we will denote by $n(x)$ the least natural number such that $y_{n(x)} \in B\left(x, \frac{1}{2} r_{x}\right)$. In this way we define the function $n: A \rightarrow \mathbb{N}$, where $\mathbb{N}$ denotes the set of natural numbers.

We show that this function is an injection. To this end fix arbitrary $x, x^{\prime} \in A$ and assume that $n(x)=n\left(x^{\prime}\right)$. This means that $y_{n(x)}=y_{n\left(x^{\prime}\right)}$. Let us put

$$
y=y_{n(x)}=y_{n\left(x^{\prime}\right)}
$$

Then we have that $y=y_{n(x)} \in B\left(x, \frac{1}{2} r_{x}\right)$ and, similarly $y=y_{n\left(x^{\prime}\right)} \in B\left(x^{\prime}, \frac{1}{2} r_{x^{\prime}}\right)$. Hence we obtain

$$
d(x, y)<\frac{1}{2} r_{x}, \quad d\left(x^{\prime}, y\right)<\frac{1}{2} r_{x^{\prime}}
$$

Consequently, we get

$$
d\left(x, x^{\prime}\right) \leqslant d(x, y)+d\left(y, x^{\prime}\right)<\frac{1}{2}\left(r_{x}+r_{x^{\prime}}\right) .
$$

Suppose that $r_{x} \leqslant r_{x^{\prime}}$. Then the above inequality implies that $d\left(x, x^{\prime}\right)<r_{x^{\prime}}$. This allows us to deduce that

$$
x \in B\left(x^{\prime}, r_{x^{\prime}}\right) \cap A=\left\{x^{\prime}\right\}
$$

which gives that $x=x^{\prime}$.

In the case when $r_{x^{\prime}} \leqslant r_{x}$, the similar reasoning leads to the same conclusion. Finally, we conclude that the function $n=n(x)$ is an injective mapping. This means that the set $A$ has the same cardinality as a certain subset of the set $\mathbb{N}$. The proof is complete.

From the above theorem we obtain the following useful corollary.

Corollary 2.5. Let $(X, d)$ be a metric space. If there exists an uncountable subset $A$ of $X$ and a number $\varepsilon>0$ such that for arbitrary $x, y \in A, x \neq y$, we have that $d(x, y) \geqslant \varepsilon$, then the space $X$ is not separable.

Proof. In view of the assumption we infer that the set $A$ is isolated. Indeed, for an arbitrary $x \in A$ we have

$$
B(x, \varepsilon) \cap A=\{x\}
$$


If $X$ would be separable then in view of Theorem 2.4 we have that $A$ is at most countable. The obtained contradiction completes the proof.

To illustrate the usefulness of Corollary 2.5 let us consider the following example.

Example 2.6. Consider the Banach sequence space $l_{\infty}$ consisting of all real bounded sequences and normed with help of the supremum norm. Then the set $A$ of all sequences with terms equal 0 or 1 is uncountable. Moreover, for $x, y \in A, x \neq y$, we have $d(x, y)=1$. Hence, in view of Corollary 2.5 we conclude that $l_{\infty}$ is not separable.

The next theorem will play a crucial role in our considerations.

Theorem 2.7. Let $A, B$ be nonempty subsets of the metric space $X$ such that $A \subset B$. Assume that for an arbitrary $x \in X$ the following condition is satisfied:

$$
x \in B \text { if and only if there exists a sequence }\left(a_{n}\right) \subset A \text { such that } a_{n} \rightarrow x .
$$

Then the set $B$ is closed and $A$ is dense in the set $B$.

Proof. Take $b \in \bar{B}$. Then there exists a sequence $\left(b_{n}\right) \subset B$ such that $b_{n} \rightarrow b$.

Since $b_{1} \in B$, we can find a sequence $\left(a_{n}^{1}\right) \subset A$ such that $a_{n}^{1} \rightarrow b_{1}$.

Further, since $b_{2} \in B$, we can find a sequence $\left(a_{n}^{2}\right) \subset A$ such that $a_{n}^{2} \rightarrow b_{2}$.

Similarly, for an arbitrary natural number $k$, taking the term $b_{k} \in B$, we can find a sequence $\left(a_{n}^{k}\right) \subset A$ such that $a_{n}^{k} \rightarrow b_{k}$ as $n \rightarrow \infty$.

Now, fix arbitrarily $\varepsilon>0$ and choose $n_{1} \in \mathbb{N}$ such that $d\left(a_{n_{1}}^{1}, b_{1}\right)<\frac{\varepsilon}{2}$. Next, we choose $n_{2} \in \mathbb{N}, n_{2}>n_{1}$, such that $d\left(a_{n_{2}}^{2}, b_{2}\right)<\frac{\varepsilon}{2}$ and so on.

Thus we can find a sequence $\left(a_{n_{k}}^{k}\right) \subset A$ such that $d\left(a_{n_{k}}^{k}, b_{k}\right)<\frac{\varepsilon}{2}$ for $k=1,2, \ldots$. Hence we got

$$
d\left(a_{n_{k}}^{k}, b\right) \leqslant d\left(a_{n_{k}}^{k}, b_{k}\right)+d\left(b_{k}, b\right)<\varepsilon
$$

for $k$ big enough. This implies that $a_{n_{k}}^{k} \rightarrow b$ if $k \rightarrow \infty$. Hence, in view of condition (D) we obtain that $b \in B$. This means that the set $B$ is closed. The conclusion that $A$ is dense in $B$ is obvious.

In the sequel of the paper we will work in the function space $B([a, b])$ consisting of all real functions defined and bounded on the interval $[a, b]$. Recall that $B([a, b])$ forms a Banach space under the supremum norm which will be denoted by $\|\cdot\|_{\infty}$ i.e., for $f \in B([a, b])$ we put

$$
\|f\|_{\infty}=\sup \{|f(x)|: x \in[a, b]\} .
$$

The space $B([a, b])$ is not separable. This space is very convenient in numerous investigations conducted in the theory of real functions and functional analysis. Let us pay our attention to two important subspaces of the space $B([a, b])$.

Namely, consider the subset $C([a, b])$ of the space $B([a, b])$ which consists of all functions continuous on the interval $[a, b]$. It is well-known that $C([a, b])$ forms a closed subset of the space $B([a, b])$ under the supremum norm. Thus $C([a, b])$ is the Banach space with the norm $\|\cdot\|_{\infty}$. 
Another important subspace of $B([a, b])$ is formed by the so-called step functions. To describe that space we introduce first the definition of the concept of a step function.

Definition 2.8. A function $f:[a, b] \rightarrow \mathbb{R}$ is called a step function if there exists a finite sequence $\left\{x_{0}, x_{1}, \ldots, x_{n}\right\} \subset[a, b]$ such that $a=x_{0}<x_{1}<x_{2}<\ldots<x_{n}=b$ and such that the function $f$ is constant on every interval $\left(x_{i-1}, x_{i}\right)(i=1,2, \ldots, n)$.

The set of all step functions on the interval $[a, b]$ will be denoted by $S([a, b])$. Let us observe that $S([a, b]) \subset B([a, b])$. Moreover, the set $S([a, b])$ is a linear space over the field of real numbers $\mathbb{R}$ with the usual operations of the addition of functions and the multiplication of a function by a real scalar. To prove this statement let us take arbitrary functions $f, g \in S([a, b])$. Then there exist two finite sets $X=$ $\left\{x_{0}, x_{1}, \ldots, x_{n}\right\}, Y=\left\{y_{0}, y_{1}, \ldots, y_{m}\right\}$ with the property $a=x_{0}<x_{1}<\ldots<x_{n}=b$, $a=y_{0}<y_{1}<\ldots<y_{m}=b$ and such that the function $f$ is constant on each interval $\left(x_{i-1}, x_{i}\right)$ for $i=1,2, \ldots, n$ and the function $g$ is constant on each interval $\left(y_{j-1}, y_{j}\right)$ for $j=1,2, \ldots, m$. Take the union $X \cup Y$ and arrange the elements of this set into an increasing sequence $Z=\left\{z_{0}, z_{1}, \ldots, z_{k}\right\}$ in such a way that if some two elements of the sets $X$ and $Y$ are the same, then we treat them as one point of the set $Z$. Thus

$$
a=z_{0}<z_{1}<\ldots<z_{k}=b .
$$

Notice that the functions $f$ and $g$ are constant on each interval $\left(z_{i-1}, z_{i}\right)$ for $i=$ $1,2, \ldots, k$. This implies that $f+g$ is also constant on each of the mentioned intervals. Thus $f+g \in S([a, b])$.

Similarly (even in an easier manner) we show that $\alpha f \in S([a, b])$ for any $\alpha \in \mathbb{R}$. Finally we conclude that $S([a, b])$ is a linear subspace of the space $B([a, b])$. This justifies our earlier assertion.

Now, we show that the space $S([a, b])$ is not complete under the norm induced from the space $B([a, b])$ i.e., under the supremum norm $\|\cdot\|_{\infty}$.

To this end let us take into account the following example.

Example 2.9. Consider the function $f \in B([0,1])$ defined in the following way

$$
f(x)= \begin{cases}\frac{1}{n+1} & \text { for } x \in\left[\frac{1}{n+1}, \frac{1}{n}\right), n=1,2, \ldots \\ 1 & \text { for } x=1 \\ 0 & \text { for } x=0 .\end{cases}
$$

Observe that $f$ is not a step function since $f$ is not constant on finite family of open subintervals of the interval $[0,1]$. Thus $f \notin S([a, b])$.

Next, let us take the sequence $\left(f_{n}\right)$ of functions defined for each fixed natural number $n$ in the following way

$$
f_{n}(x)= \begin{cases}\frac{1}{k+1} & \text { for } x \in\left[\frac{1}{k+1}, \frac{1}{k}\right), k=1,2, \ldots, n \\ 1 & \text { for } x=1 \\ 0 & \text { for } x \in\left[0, \frac{1}{n+1}\right) .\end{cases}
$$


It is easily seen that $f_{n} \in S([0,1])$ for any $n \in \mathbb{N}$. Moreover, for a fixed $n$ we have

$$
\left\|f-f_{n}\right\|_{\infty}=\frac{1}{n+2} .
$$

Hence we deduce that the sequence $\left(f_{n}\right)$ converges to the function $f$ in the topology generated by the norm of the space $B([0,1])$. Thus $f$ is a cluster point of the set $S([0,1])$. Since $f \notin S([0,1])$, we infer that the set $S([0,1])$ is not a closed set in the space $B([0,1])$. On the base of Theorem 2.2 this leads to the conclusion that the space $S([0,1])$ is not complete (under the supremum norm $\|\cdot\|_{\infty}$ ).

\section{Existence of finite limits of a function via the Cau- chy condition}

It is well-known $[3,7]$ that the Cauchy condition plays an essential role in mathematical and functional analysis. Obviously, it is very useful in the elementary theory of sequences in metric space, in the theory of series in Banach space and in the theory of real functions [7]. The fundamental importance of the concept of the Cauchy condition relies on the creating of the possibility of the defining of the completeness of a metric space.

In this section we focus on the formulation of the Cauchy condition for real functions, since this condition enables us to obtain handy tools in the theory of regulated functions.

Thus, let us assume that $D$ is a nonempty subset of the set of real numbers $\mathbb{R}$ and let $x_{0}\left(x_{0} \in \mathbb{R}\right)$ be an accumulation point of the set $D$.

Moreover, let $f: D \rightarrow \mathbb{R}$ be a given function.

Definition 3.1. We say that the function $f$ satisfies at the point $x_{0}$ the Cauchy condition if

$$
\underset{\varepsilon>0}{\forall} \underset{\delta>0}{\exists} \underset{\substack{t, s \in D \\ t \neq x_{0} \\ s \neq x_{0}}}{\forall}\left[\left|t-x_{0}\right|<\delta,\left|s-x_{0}\right|<\delta \Rightarrow|f(t)-f(s)|<\varepsilon\right] .
$$

Similarly, let us assume now that $x_{0}\left(x_{0} \in \mathbb{R}\right)$ is a left hand point of accumulation of the set $D$.

Definition 3.2. We say that the function $f: D \rightarrow \mathbb{R}$ satisfies at the point $x_{0}$ the left hand Cauchy condition if

$$
\underset{\varepsilon>0}{\forall} \underset{\delta>0}{\exists} \underset{\substack{t, s \in D \\ t<x_{0} \\ s<x_{0}}}{\forall}\left[x_{0}-t<\delta, x_{0}-s<\delta \Rightarrow|f(t)-f(s)|<\varepsilon\right] .
$$

In the same way we can formulate the definition of the concept of the right hand Cauchy condition. Namely, assume that $x_{0}\left(x_{0} \in \mathbb{R}\right)$ is a right hand point of accumulation of the set $D$. 
Definition 3.3. We say that the function $f: D \rightarrow \mathbb{R}$ satisfies at the point $x_{0}$ the right hand Cauchy condition if

$$
\underset{\varepsilon>0}{\forall} \underset{\delta>0}{\exists} \underset{\substack{t, s \in D \\ t>x_{0} \\ s>x_{0}}}{\forall}\left[t-x_{0}<\delta, s-x_{0}<\delta \Rightarrow|f(t)-f(s)|<\varepsilon\right] .
$$

It is well known $[7,11]$ that the existence of a finite limit of the function $f: D \rightarrow \mathbb{R}$ is equivalent to the Cauchy condition. We formulate this result in details.

Theorem 3.4. Assume that $D$ is a nonempty subset of the set $\mathbb{R}$ and $x_{0}\left(x_{0} \in \mathbb{R}\right)$ is an accumulation point of the set $D$ (a left hand accumulation point of $D$; a right hand accumulation point of $D$, respectively). Let $f: D \rightarrow \mathbb{R}$ be a given function. Then:

(i) The finite limit $\lim _{x \rightarrow x_{0}} f(x)$ does exist if and only if the function $f$ satisfies the Cauchy condition at the point $x_{0}$.

(ii) The finite left hand limit $\lim _{x \rightarrow x_{0}^{-}} f(x)$ does exist if and only if the function $f$ satisfies the left hand Cauchy condition at the point $x_{0}$.

(iii) The finite right hand limit $\lim _{x \rightarrow x_{0}^{+}} f(x)$ does exist if and only if the function $f$ satisfies the right hand Cauchy condition at the point $x_{0}$.

Remark 3.5. Observe that the Cauchy condition for the function $f: D \rightarrow \mathbb{R}$ can be also formulated in the case when we assume that $-\infty$ or $+\infty$ is the accumulation point of the set $D$. Then we can also formulate a suitable version on the existence of finite limits of the function $f$ at $-\infty$ or at $+\infty$, similarly as in the case of Theorem 3.4. We omit details.

\section{Regulated functions and their properties}

In this section we will discuss the concept of a regulated function. To make our presentation more transparent we restrict ourselves to real functions i.e., to functions with values in the set of real numbers $\mathbb{R}$.

The possible generalization to the case of functions with values in an arbitrary Banach space will be discussed in the next section.

Definition 4.1. A function $f \in B([a, b])$ is called a regulated function if it has onesided limits at every point $x \in(a, b)$ and if it has the right hand limit at $x=a$ and the left hand limit at $x=b$.

Other words, $f$ is regulated on the interval $[a, b]$ if for each $x \in(a, b)$ there exist limits $f(x-), f(x+)$ and there exist limits $f(a+), f(b-)$.

The class of all regulated functions on the interval $[a, b]$ will be denoted by $R([a, b])$.

Observe that the assumption requiring that $f$ is a member of the space $B([a, b])$ implies that the limits indicated in Definition 4.1 are finite. 
Now, we show that the concept of a regulated function can be presented in other way if we dispense with the assumption that $f \in B([a, b])$.

Indeed, we have the following theorem.

Theorem 4.2. Let $f:[a, b] \rightarrow \mathbb{R}$ be a function with the property that for each $x_{0} \in[a, b]$ there exist finite one-sided limits $f\left(x_{0}-\right)$ and $f\left(x_{0}+\right)$ of the function $f$ at the point $x_{0}$ (in the case $x_{0}=a$ we assume that there exists the finite one-sided limit $f(a+)$ and similarly, there exists a finite one-sided limit $f(b-)$ in the case $\left.x_{0}=b\right)$. Then the function $f$ is bounded on the interval $[a, b]$.

Proof. Suppose contrary i.e., the function $f$ is not bounded on the interval $[a, b]$. In order to fix our attention let us assume that $f$ is not bounded from above on $[a, b]$. Then there exists a sequence $\left(x_{n}\right) \subset[a, b]$ such that $f\left(x_{n}\right) \geqslant n$ for $n=1,2, \ldots$ Since the sequence $\left(x_{n}\right)$ is bounded, in view of Bolzano-Weierstrass theorem we infer that there exists a subsequence $\left(x_{k_{n}}\right)$ converging to some point $x_{0} \in[a, b]$. Then we have

$$
f\left(x_{k_{n}}\right) \geqslant k_{n}
$$

for $n=1,2, \ldots$. Observe that we can assume that we can select the subsequence $\left(x_{k_{n}}\right)$ in such a way that $x_{k_{n}} \neq x_{0}$ for $n=1,2, \ldots$. If this would be not possible then $x_{k_{n}} \neq x_{0}$ only for finite number of terms of the sequence $\left(x_{k_{n}}\right)$ i.e., there would be exist a subsequence $\left(x_{p_{n}}\right)$ of the sequence $\left(x_{k_{n}}\right)$ such that $x_{p_{n}}=x_{0}$ for $n=1,2, \ldots$. Then

$$
f\left(x_{p_{n}}\right)=f\left(x_{0}\right) \geqslant p_{n}
$$

for $n=1,2, \ldots$. Hence, taking into account that $p_{n} \rightarrow \infty$ for $n \rightarrow \infty$, we infer that $f\left(x_{0}\right)=\infty$.

The obtained contradiction shows that we can assume that $x_{k_{n}} \neq x_{0}$ for $n=1,2, \ldots$.

Next, let us notice that we can select a subsequence $\left(x_{l_{n}}\right)$ of the sequence $\left(x_{k_{n}}\right)$ such that $x_{l_{n}}<x_{0}$ for $n=1,2, \ldots$ or, we can select a subsequence $\left(x_{q_{n}}\right)$ of $\left(x_{k_{n}}\right)$ such that $x_{q_{n}}>x_{0}$ for $n=1,2, \ldots$. Obviously, both sequences $\left(x_{l_{n}}\right)$ and $\left(x_{q_{n}}\right)$ are converging to $x_{0}$. In order to fix our attention let us assume that there exists a subsequence $\left(x_{l_{n}}\right)$ of the sequence $\left(x_{k_{n}}\right)$ such that $x_{l_{n}}<x_{0}(n=1,2, \ldots)$ and $x_{l_{n}} \rightarrow x_{0}$. Then we have

$$
f\left(x_{l_{n}}\right) \geqslant l_{n}
$$

for $n=1,2, \ldots$. Hence we get that

$$
\lim _{n \rightarrow \infty} f\left(x_{l_{n}}\right)=\infty
$$

But this contradicts to the assumption that there exists a finite left hand limit $f\left(x_{0}-\right)$. Obviously in the case $x_{0}=a$ or $x_{0}=b$ the proof is similar.

The above theorem shows that in the definition of the regulated function $f$ instead of the assumption that $f \in B([a, b])$ we can equivalently assume that the function $f:[a, b] \rightarrow \mathbb{R}$ has finite one-sided limits at every point of the interval $[a, b]$. 
In what follows let us note that we have the inclusion $R([a, b]) \subset B([a, b])$ which follows immediately from Definition 4.1. Further, observe that $S([a, b]) \subset R([a, b])$ which is a simple consequence of the definitions of a step function and a regulated function. Obviously, the converse inclusion is not valid. Indeed, let us consider again Example 2.9. Then we see that the function $f$ considered in that example is regulated but it is not a step function.

Now, we show that the set $R([a, b])$ forms a linear subspace of the space $B([a, b])$. To prove this fact take arbitrary functions $f, g \in R([a, b])$ and fix a point $x_{0} \in$ $(a, b)$. Then there exist the limits $f\left(x_{0}-\right), f\left(x_{0}+\right), g\left(x_{0}-\right), g\left(x_{0}+\right)$. By the standard theorems of mathematical analysis we infer that

$(f+g)\left(x_{0}-\right)=\lim _{x \rightarrow x_{0}-}(f(x)+g(x))=\lim _{x \rightarrow x_{0}-} f(x)+\lim _{x \rightarrow x_{0}-} g(x)=f\left(x_{0}-\right)+g\left(x_{0}-\right)$.

Similarly we show that there exist (finite) limits $(f+g)\left(x_{0}+\right)$ and $(f+g)(a+),(f+$ $g)(b-)$.

Thus $f+g \in R([a, b])$.

The proof showing that $\alpha \cdot f \in R([a, b])$ for $\alpha \in \mathbb{R}$ is also standard.

Thus the set $R([a, b])$ of regulated functions on the interval $[a, b]$ has the algebraic structure of a linear space over the field $\mathbb{R}$.

Moreover, the space $R([a, b])$, as a subspace of the Banach space $B([a, b])$, forms a normed space with respect to the supremum norm $\|\cdot\|_{\infty}$. However, the proof of the fact, that this norm is complete (i.e., that $R([a, b])$ is a Banach space under the norm $\left.\|\cdot\|_{\infty}\right)$, is not easy [3].

The key role in the announced proof is played by the following theorem.

Theorem 4.3. Let $f \in B([a, b])$. Then $f \in R([a, b])$ if and only if there exists a sequence $\left(f_{n}\right)$ of step functions on the interval $[a, b]\left(i . e .,\left(f_{n}\right) \subset S([a, b])\right)$ such that it is uniformly convergent on the interval $[a, b]$ to the function $f$ (equivalently: $\lim _{n \rightarrow \infty} \| f_{n}-$ $\left.f \|_{\infty}=0\right)$.

Proof. At first, we prove the part $\Rightarrow$.

Thus, take a function $f \in R([a, b])$. Fix arbitrarily a natural number $n$ and $x \in[a, b]$. Assume first that $x \in(a, b)$. Then, taking into account Theorem 3.4 we deduce that there exists an interval $(y(x), z(x)) \subset[a, b]$ such that $x \in(y(x), z(x))$ and for arbitrary $t, s \in(y(x), x)$ we have that $|f(t)-f(s)| \leqslant \frac{1}{n}$ and, for arbitrary $t, s \in(x, z(x))$ we have that $|f(t)-f(s)| \leqslant \frac{1}{n}$.

Similarly, taking $x=a$ we can meet an interval $[a, z(a)) \subset[a, b]$ such that for $t, s \in$ $(a, z(a))$ we have $|f(t)-f(s)| \leqslant \frac{1}{n}$. In the same way we find an interval $(y(b), b] \subset[a, b]$ such that $|f(t)-f(s)| \leqslant \frac{1}{n}$ for $t, s \in(y(b), b)$.

Further, consider the following family of the intervals:

$$
\{(y(x), z(x))\}_{x \in(a, b)} \cup\{[a, z(a)),(y(b), b]\} .
$$

This family forms an open covering of the interval $[a, b]$ (in the topological space formed by the interval $[a, b]$ with the natural metric). Since the space $[a, b]$ is compact 
we can select a finite family of intervals

$$
\left\{\left(y\left(x_{i}\right), z\left(x_{i}\right)\right)\right\}_{1 \leqslant i \leqslant m} \cup\{[a, z(a)),(y(b), b]\},
$$

which is a subcovering of the interval $[a, b]$.

Next, let $\left(c_{j}\right)_{0 \leqslant j \leqslant k}$ be a strictly increasing finite sequence formed by the numbers $a, b, z(a), y(b), x_{i}, y\left(x_{i}\right), z\left(x_{i}\right)(i=1,2, \ldots, m)$. Obviously $c_{0}=a, c_{k}=b$.

Observe that each of the intervals $\left(c_{0}, c_{1}\right),\left(c_{1}, c_{2}\right), \ldots,\left(c_{k-1}, c_{k}\right)$ is located in a certain of the selected intervals which form a finite covering of $[a, b]$. On the other hand excluding the first and the last interval, any of such an interval is contained either in an interval $\left(y\left(x_{i}\right), x_{i}\right)$ or in an interval $\left(x_{i}, z\left(x_{i}\right)\right)$. Similar assertion holds for the intervals $\left[c_{0}, c_{1}\right),\left(c_{k-1}, c_{k}\right]$. Thus, for arbitrary $t, s \in\left(c_{j-1}, c_{j}\right)(j=1,2, \ldots, k)$ we have that $|f(t)-f(s)| \leqslant \frac{1}{n}$.

Now, for an arbitrarily fixed $j \in\{1,2, \ldots, k\}$, let us define a function $f_{n}$ to be equal to the value of the function $f$ at an arbitrary point in the interval $\left(c_{j-1}, c_{j}\right)$. Moreover, we put $f_{n}\left(c_{j}\right)=f\left(c_{j}\right)$ for $j=0,1, \ldots, k$.

In this way we obtain a step function $f_{n}$ such that

$$
\left\|f_{n}-f\right\|_{\infty} \leqslant \frac{1}{n}
$$

for $n=1,2, \ldots$. Hence we obtain that $\lim _{n \rightarrow \infty}\left\|f_{n}-f\right\|_{\infty}=0$.

Now, we are going to prove the implication $\Leftarrow$.

To this end assume that $f \in B([a, b])$ is a function being the limit of uniformly convergent sequence $\left(f_{n}\right)$ of step functions on the interval $[a, b]$. Fix an arbitrary number $\varepsilon>0$. Then we can find a natural number $n$ such that

$$
\left\|f_{n}-f\right\|_{\infty} \leqslant \frac{\varepsilon}{3} .
$$

Keeping in mind the fact that $f_{n}$ is a step function we deduce that for each $x \in(a, b)$ there exists an interval $(c, d)$ containing $x$ (and, for $x=a$, there exists an interval $(a, c)$, whereas for $x=b$ there exists an interval $(d, b))$ such that

$$
\left|f_{n}(t)-f_{n}(s)\right| \leqslant \frac{\varepsilon}{3}
$$

if $t, s \in(c, x)$ or if $t, s \in(x, d)$ (the situation is even simpler in the case of end intervals).

Further, let us take an arbitrary number $x \in[a, b]$ and next, let us choose an interval $(c, x)$ or $(x, d)$ such that for $t, s \in(c, x)$ (or for $t, s \in(x, d))$ the inequality (4.2) is satisfied.

In order to fix our attention assume that $(c, x)$ is the desired interval. Then, for $t, s \in(c, x)$, in view of (4.1) and (4.2) we get

$$
|f(t)-f(s)| \leqslant\left|f(t)-f_{n}(t)\right|+\left|f_{n}(t)-f_{n}(s)\right|+\left|f_{n}(s)-f(s)\right| \leqslant \frac{\varepsilon}{3}+\frac{\varepsilon}{3}+\frac{\varepsilon}{3}=\varepsilon .
$$


From the above inequality and Theorem 3.4 we conclude that there exists the left hand limit of the function $f$ at the point $x$.

In the similar way we show that there exists the right hand limit $f(x+)$.

This means that $f \in R([a, b])$.

Finally, taking into account the above proved theorem and Theorem 2.7 we infer that $R([a, b])$ is a closed subset of the space $B([a, b])$. Since the space $B([a, b])$ is complete, on the base of Theorem 2.1 we deduce that the space of regulated functions $R([a, b])$ is complete.

We formulate the above statement as the separated theorem.

Theorem 4.4. The space of regulated functions $R([a, b])$ with the supremum norm is a Banach space.

Observe that the space $R([a, b])$ is not separable. Indeed, fix arbitrarily $\varepsilon>0$. For a fixed number $y \in[a, b]$ define the function $f_{y}$ in the following way

$$
f_{y}(x)= \begin{cases}\varepsilon & \text { for } x=y \\ 0 & \text { for } x \in[a, b], x \neq y\end{cases}
$$

Obviously $f_{y} \in R([a, b])$. Further notice that for $y_{1}, y_{2} \in[a, b], y_{1} \neq y_{2}$, we have that $\left\|f_{y_{1}}-f_{y_{2}}\right\|_{\infty}=\varepsilon$. Hence, by virtue of Corollary 2.5 we obtain our claim. In what follows we pay our attention to points of discontinuity of regulated functions. At the beginning we recall the classification of points of discontinuity accepted in mathematical analysis $[1,7,11]$.

Assume that $f:[a, b] \rightarrow \mathbb{R}$ is a given function.

Definition 4.5. Let $x_{0} \in[a, b]$ be a point of discontinuity of the function $f$. We say that $x_{0}$ is a point of discontinuity of the first kind if there exist finite one-sided limits $f\left(x_{0}-\right)=\lim _{x \rightarrow x_{0}-} f(x), f\left(x_{0}+\right)=\lim _{x \rightarrow x_{0}+} f(x)$. In the case $x_{0}=a$ or $x_{0}=b$ we assume the existence of finite limits $f(a+), f(b-)$, but it has to be satisfied the condition $f(a+) \neq f(a), f(b-) \neq f(b)$, respectively.

Observe that if $x_{0}$ is a point of discontinuity of the first kind then the following situations may occur:

$1^{\circ} f\left(x_{0}-\right) \neq f\left(x_{0}+\right)$.

In this case the point of discontinuity of the function $f$ is called a jump.

$2^{\circ} f\left(x_{0}-\right)=f\left(x_{0}+\right) \neq f\left(x_{0}\right)$.

In such a case we will say that $f$ has at the point $x_{0}$ a removable discontinuity.

Definition 4.6. A point of discontinuity $x_{0}$ is called a point of discontinuity of the second kind if it is not a point of discontinuity of the first kind i.e., at least one of the one-sided limits is unbounded or does not exist.

Now, let us observe that if $f \in B([a, b])$ then $f$ may have points of discontinuity of the first kind only. This implies that a regulated function on the interval $[a, b]$ has 
only points of discontinuity of the first kind. Thus, assume that $f \in R([a, b])$. Let us accept the following notation [1]:

$$
\begin{aligned}
D(f) & =\{x \in[a, b]: f \text { is discontinuous at } x\}, \\
D_{0}(f) & =\{x \in[a, b]: f \text { has a removable discontinuity at } x\}, \\
D_{1}(f) & =\{x \in[a, b]: f \text { has a jump at } x\} .
\end{aligned}
$$

Observe that if $a \in D(f)(b \in D(f))$ then $a \in D_{0}(f)\left(b \in D_{0}(f)\right)$.

It is worthwhile mentioning that if $f$ is a monotone function on the interval $[a, b]$ then $f$ has only points of discontinuity being jumps i.e., $D(f)=D_{1}(f)$. On the other hand it is well known that the set of points of discontinuity of each function which is monotone on the interval $[a, b]$ is at most countable.

The similar result holds also for any function $f \in R([a, b])$. Indeed, we have the following theorem [1].

Theorem 4.7. The set of all points of discontinuity of any regulated function on the interval $[a, b]$ is at most countable.

The proof of this result is given in [1] and we will not repeat it in this paper. However, we provide the proof of an analogous result in the next section for regulated functions on the interval $[a, b]$ with values in an arbitrary separable Banach space.

At the end of this section we provide a few facts indicating some special classes of regulated functions.

First of all let us pay our attention to the class of functions of bounded variation on the interval $[a, b]$ (cf. [1]). Let us denote this class by $B V([a, b])$.

Notice that $S([a, b]) \subset B V([a, b]) \subset B([a, b])$. Similarly as in the case of step functions it is easily seen that $B V([a, b])$ is a linear space over the field $\mathbb{R}$ (cf. [1]). Moreover, the space $B V([a, b])$ is not complete with respect to the supremum norm $\|\cdot\|_{\infty}$.

Further, let us pay attention to the fact that by the classical Jordan decomposition theorem we have that $B V([a, b]) \subset R([a, b])$.

The next important generalization of the space $B V([a, b])$ is the class of functions having the so-called bounded Wiener variation of order $p$ (cf. [1]).

To define this class, fix a number $p \in[1, \infty)$. For a partition $P=\left\{x_{0}, x_{1}, \ldots, x_{m}\right\}$ of the interval $[a, b]$, where $a=x_{0}<x_{1}<\ldots<x_{m}=b$, we define the Wiener variation (of order $p$ ) of a function $f \in B([a, b])$ with respect to the partition $P$ by putting

$$
\operatorname{Var}_{p}^{W}(f, P ;[a, b])=\sum_{i=1}^{m}\left|f\left(x_{i}\right)-f\left(x_{i-1}\right)\right|^{p} .
$$

The Wiener variation of order $p$ of $f$ is defined as

$$
\operatorname{Var}_{p}^{W}(f)=\operatorname{Var}_{p}^{W}(f ;[a, b])=\sup \left\{\operatorname{Var}_{p}^{W}(f, P ;[a, b]): P \in \mathcal{P}([a, b])\right\},
$$

where the symbol $\mathcal{P}([a, b])$ stands for the set of all partitions of the interval $[a, b]$. A function $f(f \in B([a, b]))$ is called of bounded $p$-th variation if $\operatorname{Var}_{p}^{W}(f)<\infty$. 
By the symbol $W B V_{p}([a, b])$ we denote the class of all functions of bounded Wiener $p$-th variation.

It can be shown [1] that $W B V_{p}([a, b])$ is a linear space and $B V([a, b]) \subset$ $W B V_{p}([a, b]) \subset R([a, b])$ for any $p \geqslant 1$, but there are regulated functions which do not belong to the space $W B V_{p}([a, b])$.

In order to introduce the next class of functions denote by $\phi$ the so-called Young function i.e., $\phi: \mathbb{R}_{+} \rightarrow \mathbb{R}_{+}, \phi(0)=0, \phi(t)>0$ for $t>0, \phi$ is continuous and convex on $\mathbb{R}_{+}$. Then, we can define the Wiener-Young variation of a function $f \in B([a, b])$ with respect to a partition $\mathcal{P}$ of the interval $[a, b]$ by putting

$$
\operatorname{Var}_{\phi}^{W}(f, P)=\sum_{i=1}^{m} \phi\left(\left|f\left(x_{i}\right)-f\left(x_{i-1}\right)\right|\right) \text {. }
$$

The formula

$$
\operatorname{Var}_{\phi}^{W}(f)=\sup \left\{\operatorname{Var}_{\phi}^{W}(f, P): P \in \mathcal{P}([a, b])\right\}
$$

defines the Wiener-Young variation of $f$ on the interval $[a, b]$.

If we denote by $W B V_{\phi}([a, b])$ the class of functions of bounded Wiener-Young variation on $[a, b]$ then it can be shown that $W B V_{\phi}([a, b]) \subset R([a, b])$ and

$$
\bigcup_{\phi \in \Phi} W B V_{\phi}([a, b])=R([a, b])
$$

where $\Phi$ denotes the class of all Young functions $[8,9]$.

\section{Generalizations}

This section is devoted to show that the concept of a regulated function can be introduced in a more general setting.

Namely, let $(E,\|\cdot\|)$ be a real Banach space. We will denote by $B(x, r)$ the open ball in $E$ centered at $x$ and with radius $r$. The symbol $B([a, b], E)$ will denote the class of all functions acting from $[a, b]$ into $E$ which are bounded on $[a, b]$. Obviously $B([a, b], E)$ forms a linear space and it can be equipped with the classical supremum norm $\|\cdot\|_{\infty}$ defined for $f \in B([a, b], E)$ in the following way

$$
\|f\|_{\infty}=\sup \{\|f(x)\|: x \in[a, b]\} .
$$

The space $B([a, b], E)$ is a Banach space.

Similarly, we can define the Banach space $C([a, b], E)$ consisting of all functions defined and continuous on the interval $[a, b]$ with values in $E$ and with supremum norm $\|\cdot\|_{\infty}$.

Finally, let us consider the set $R([a, b], E)$ consisting of all regulated functions $f$ : $[a, b] \rightarrow E$. In other words, $f \in R([a, b], E)$ if $f \in B([a, b], E)$ and the function $f$ has one-sided limits at every point $x \in(a, b)$ and if it has the right hand limit $f(a+)$ and 
the left hand limit $f(b-)$.

In the same way as in Section 4 we can show that the set $R([a, b], E)$ forms a linear space with usual operations on functions. Moreover, if we introduce in $R([a, b], E)$ the supremum norm $\|\cdot\|_{\infty}$ then we can prove that $R([a, b], E)$ is a Banach space. The proof of this theorem can be conducted exactly in the same way as the proof of Theorem 4.4 and, similarly as before, the space of step functions $S([a, b], E)$ plays the key role in argumentation of that proof. Therefore, we will not repeat details of the proof.

In this section we restrict ourselves to prove an analogon of Theorem 4.7 in the case of the space of regulated functions $R([a, b], E)$. To adopt some reasonings utilized in the proof of Theorem 4.7 given in [1], we will assume that the Banach space $E$ is separable. Nevertheless, we provide here the details of the complete proof of the announced theorem to fill in some gaps occuring in the suitable proof given in [1].

Theorem 5.1. Let $E$ be a separable Banach space. Then the set of all points of discontinuity of an arbitrary regulated function $f \in R([a, b], E)$ is at most countable.

Proof. Observe that we can restrict ourselves to the set of points of discontinuity of $f$ belonging to the interval $(a, b)$, since the set of points of discontinuity of $f$ on the interval $[a, b]$ can differ only of at most two points. Further, consider the average function $\bar{f}$ of $f$ defined in the following way

$$
\bar{f}(x)= \begin{cases}\frac{1}{2}(f(x-)+f(x+)) & \text { for } x \in D_{1}(f) \\ f(x) & \text { otherwise. }\end{cases}
$$

Obviously we have that $D(\bar{f})=D(f), D_{0}(\bar{f})=D_{0}(f), D_{1}(\bar{f})=D_{1}(f)$, where the sets $D(f), D_{0}(f)$ and $D_{1}(f)$ were defined earlier. Thus, it is sufficient to show that the set $D(\bar{f})$ is at most countable.

To this end assume first that $x_{0} \in D_{0}(f)$. Then $f\left(x_{0}-\right)=f\left(x_{0}+\right) \neq f\left(x_{0}\right)$. Take the number

$$
\varepsilon=\frac{1}{2}\left\|f\left(x_{0}\right)-f\left(x_{0}+\right)\right\| .
$$

Taking into account the definition of the one-sided limit of a function we can find a number $\delta>0$ such that for $x \in\left(x_{0}-\delta, x_{0}\right)$ we have

$$
\left\|f(x)-f\left(x_{0}-\right)\right\|<\varepsilon
$$

and for $x \in\left(x_{0}, x_{0}+\delta\right)$ we have

$$
\left\|f(x)-f\left(x_{0}+\right)\right\|<\varepsilon .
$$

Now, let us observe that for $x \in\left(x_{0}-\delta, x_{0}\right) \cup\left(x_{0}, x_{0}+\delta\right)$ the following inequality holds

$$
\left\|f(x)-f\left(x_{0}\right)\right\|>\varepsilon
$$


To prove this inequality suppose contrary. Then, for some $x \in\left(x_{0}-\delta, x_{0}\right) \cup\left(x_{0}, x_{0}+\delta\right)$ we have

$$
\left\|f(x)-f\left(x_{0}\right)\right\| \leqslant \varepsilon .
$$

Now, in view of (5.1), (5.2) and (5.4), we get

$$
\left.\left\|f\left(x_{0}\right)-f\left(x_{0}+\right)\right\| \leqslant \| f\left(x_{0}\right)-f(x)\right)\|+\| f(x)-f\left(x_{0}+\right) \|<2 \varepsilon,
$$

which contradicts to the choice of the number $\varepsilon$.

This shows that in the ball $B\left(\left(x_{0}, f\left(x_{0}\right)\right), r\right)$ in the Banach space $[a, b] \times E$ (with the maximum norm, for example), where $r=\min \{\delta, \varepsilon\}$, there are no points of the graph of the function $f$ except the point $\left(x_{0}, f\left(x_{0}\right)\right)$. Thus the point $\left(x_{0}, f\left(x_{0}\right)\right)$ is an isolated point of the graph of the function $f$ ( or $\bar{f}$ ).

Now, assume that $x_{0} \in D_{1}(f)$. Then $f\left(x_{0}-\right) \neq f\left(x_{0}+\right)$. Let us put $\varepsilon=\frac{1}{4} \| f\left(x_{0}+\right)-$ $f\left(x_{0}-\right) \|$. Taking into account the definition of one-sided limits let us find the number $\delta>0$ such that for $x \in\left(x_{0}-\delta, x_{0}\right)$ the following inequality is satisfied

$$
\left\|f(x)-f\left(x_{0}-\right)\right\|<\varepsilon,
$$

while for $x \in\left(x_{0}, x_{0}+\delta\right)$ we have

$$
\left\|f(x)-f\left(x_{0}+\right)\right\|<\varepsilon .
$$

Further, observe that for $x \in\left(x_{0}-\delta, x_{0}\right) \cup\left(x_{0}, x_{0}+\delta\right)$ the following inequality holds

$$
\left\|f(x)-\bar{f}\left(x_{0}\right)\right\|>\varepsilon \text {. }
$$

Indeed, suppose contrary. Then, for some $x \in\left(x_{0}-\delta, x_{0}\right) \cup\left(x_{0}, x_{0}+\delta\right)$ we have

$$
\left\|f(x)-\bar{f}\left(x_{0}\right)\right\| \leqslant \varepsilon .
$$

Then, linking inequalities (5.5), (5.6) and (5.8) we get

$$
\left\|\bar{f}\left(x_{0}\right)-f\left(x_{0}+\right)\right\| \leqslant\left\|\bar{f}\left(x_{0}\right)-f(x)\right\|+\left\|f(x)-f\left(x_{0}+\right)\right\|<\varepsilon+\varepsilon=2 \varepsilon .
$$

On the other hand, we have

$\left\|\bar{f}\left(x_{0}\right)-f\left(x_{0}+\right)\right\|=\left\|\frac{1}{2}\left(f\left(x_{0}-\right)+f\left(x_{0}+\right)\right)-f\left(x_{0}+\right)\right\|=\frac{1}{2}\left\|f\left(x_{0}-\right)-f\left(x_{0}+\right)\right\|=2 \varepsilon$.

Thus we obtain the contradiction with inequality (5.9). This proves inequality (5.7).

Further, consider the ball $B\left(\left(x_{0}, \bar{f}\left(x_{0}\right)\right), r\right)$ in the space $[a, b] \times E$ (considered earlier), where $r=\min \{\delta, \varepsilon\}$. Then from inequality (5.7) we conclude that this ball contains no points of the graph of the function $\bar{f}$ except the point $\left(x_{0}, \bar{f}\left(x_{0}\right)\right)$. Hence we deduce that $\left(x_{0}, \bar{f}\left(x_{0}\right)\right)$ is an isolated point of the function $\bar{f}$.

Summing up, we proved that the set $H$ of all points of the graph of the function $\bar{f}$, which are the centers of the described balls, consists of only isolated points. Thus, according to the earlier given definition, the set $H$ is an isolated set in the space $[a, b] \times E$ with the maximum norm. Obviously the mentioned space is separable. Hence, in view of Theorem 2.4 we infer that the set $H$ is at most countable. This leads to the final conclusion that the set $D(\bar{f})$ (and the set $D(f)$ ) is at most countable. 


\section{References}

[1] J. Appell, J. Banaś and N. Merentes, Bounded Variation and Around, Series in Nonlinear Analysis and Applications 17, De Gruyter, Berlin 2014.

[2] G. Aumann, Reelle Funktionen, Springer Verlag, Berlin 1954.

[3] J. Dieudonné, Foundations of Modern Analysis, Academic Press, New York 1969.

[4] D. Fraňková, Regulated functions, Math. Bohemica 116 (1991) 20-59.

[5] C. Goffman, G. Moran and D. Waterman, The structure of regulated functions, Proc. Amer. Math. Soc. 57 (1976) 61-65.

[6] R. Eochowski, R. Ghomrasni, The play operator, the truncated variation and the generalization of the Jordan decomposition, Math. Appl. Sci. 38 (2015) 403-419.

[7] S. Eojasiewicz, An Introduction to the Theory of Real Functions, J. Wiley and Sons, Chichester 1988.

[8] F. Prus-Wiśniowski, Some remarks on functions of bounded $\varphi$-variation, Ann. Soc. Math. Pol., Comment. Math. 30 (1990) 147-166.

[9] F. Prus-Wiśniowski, Continuous functions of bounded $\varphi$-variation, Ann. Soc. Math. Pol., Comment. Math. 31 (1991) 127-146.

[10] W. Rudin, Principles of Mathematical Analysis, McGraw Hill, New York 1964.

[11] R. Sikorski, Funkcje Rzeczywiste, PWN, Warsaw 1958 (in Polish).

\section{DOI: $10.7862 /$ rf.2017.2}

\section{Józef Banaś}

email: jbanas@prz.edu.pl

Department of Nonlinear Analysis

Rzeszów University of Technology

al. Powstańców Warszawy 8

35-959 Rzeszów

POLAND

\section{Mariola Kot}

email: m_kot@prz.edu.pl

Department of Nonlinear Analysis

Rzeszów University of Technology

al. Powstańców Warszawy 8

35-959 Rzeszów

POLAND

Received 2.12.2016 